\title{
Dominios de provisión de servicios en fonoaudiología y Atención Primaria de Salud a propósito de la COVID-19: Una revisión narrativa
}

\author{
Nicole Lobos Villatoro ${ }^{\mathbf{a}, *}$, Manuel del Campo Rivas ${ }^{\mathbf{b}}$, Angélica Silva-Rios ${ }^{\mathbf{b}}$ \\ a Programa Intervención Comunitaria, Universidad de Las Américas, Chile \\ ${ }^{\mathbf{b}}$ Escuela de Fonoaudiología, Facultad de Salud, Universidad Santo Tomás, Chile
}

\section{RESUMEN}

La pandemia por COVID-19 ha desafiado a los equipos profesionales en cuanto a la provisión de servicios sanitarios en Atención Primaria de Salud (APS). Los fonoaudiólogos que se desempeñan en este nivel han diversificado sus quehaceres, por lo que se vuelve necesario reflexionar sobre la práctica en este contexto. El objetivo de esta revisión narrativa es actualizar el estado del arte respecto a los dominios de la provisión de servicios en fonoaudiología y la pertinencia de los mismos en APS, a propósito de la pandemia. Los diversos ámbitos de actuación fonoaudiológica resultan atingentes para afrontar las demandas derivadas de la pandemia en APS. Existe una ampliación del quehacer fonoaudiológico que considera la continuidad del cuidado y el acompañamiento terapéutico. Dicha ampliación tiene como fin fortalecer las acciones de prevención y promoción de la salud, considerando la comunicación como una herramienta para cumplir con este fin. En este contexto, se requiere reflexionar sobre el papel disciplinar del fonoaudiólogo desde su práctica en ámbitos genéricos y específicos a nivel local, reconociendo la existencia de una hibridación en la provisión de servicios tanto a nivel individual como colectivo. También, se vuelve necesaria la formulación de documentos de posición, basados en la evidencia y sustentados en recomendaciones internacionales, así como en los requerimientos de la comunidad, respecto al rol del fonoaudiólogo en APS. La formulación y difusión de investigaciones que visibilicen los aportes de la Fonoaudiología en este ámbito parecen fundamentales.

\section{Speech and language practice domains in Primary Health Care regarding the COVID-19: A narrative review}

\section{ABSTRACT}

COVID-19 pandemic has challenged professional teams regarding the provision of health services in Primary Health Care (PHC). Speech pathologists who work at this level have diversified their work, so it becomes necessary to reflect on their practice in this context. The objective of this narrative review is to update the state of the art regarding the domains of the provision of services in speech therapy and their relevance in PHC, regarding the pandemic. The various areas of speech therapy action are pertinent to face the demands derived from the pandemic in PHC. There is an extension of scope of speech therapy practice that considers the continuity of care and therapeutic accompaniment. Such an extension incorporates the need to strengthen prevention and health promotion actions, observing communication as a tool for this purpose. In this context, it is necessary to reflect on the disciplinary role of the speech therapist from his practice in generic and specific areas at the local level, recognizing the existence of a hybridization in the provision of services both individually and collectively. It becomes also necessary to formulate position papers, based on evidence and supported by international recommendations as well as the requirements of the community, regarding the role of the speech therapist in PHC. The formulation and dissemination of research that make visible the contributions of speech therapy in this area seem fundamental.

\section{Palabras clave:}

Fonoaudiología; Atención Primaria de Salud; COVID-19

\section{Keywords:}

Speech language and hearing sciences; Primary Health Care; COVID-19 
La enfermedad por Coronavirus (COVID-19), asociada a un síndrome respiratorio agudo de carácter severo (Sheehy, 2020) fue reportada por primera vez el mes de diciembre de 2019 en la ciudad de Wuhan, China (Rothan \& Byrareddy, 2020). La rápida diseminación del virus, con un número reproductivo básico (R0) promedio de 3,28 y una mediana de 2,79 (Liu et al., 2020), generó la declaración de pandemia por parte de la Organización Mundial de la Salud (OMS) el día 11 de marzo de 2020 (Cucinotta \& Vanelli, 2020). En Chile el primer caso confirmado de COVID19, apareció el 3 de marzo del año 2020 en el Hospital Regional de la Ciudad de Talca (Ministerio de Salud de Chile [MINSAL], 2020a). El 12 de Julio del mismo año se registraron en el país 356.695 casos acumulados (MINSAL, 2020b). A nivel mundial el 16 de julio se informaron 13.530 .628 casos y 583.892 muertes (Europe Centre for Disease Prevention and Control [ECDC], 2020).

Actualmente, la estrategia del Ministerio de Salud chileno (MINSAL) para enfrentar la pandemia, tiene como eje las acciones de vigilancia epidemiológica vinculadas al testeo, trazabilidad y aislamiento (TTA) (MINSAL, 2020c), lo que ha relevado el papel de la Atención Primaria de Salud (APS). Según el tercer informe de monitoreo de la estrategia de TTA (Colegio Médico de Chile [COLMED], 2020a), un 76\% de los centros informantes realiza testeo activo y un $32 \%$ de los mismos es capaz de aislar a los casos en un tiempo inferior a 24 horas. A nivel internacional se destaca la urgencia de fortalecer la APS (Souza et al., 2020), ya que representa un pilar fundamental para la contención y manejo de la enfermedad por COVID-19 (AguilarGuerra \& Reed, 2020).

En Chile el Estatuto de Salud Primaria de Atención Municipal norma la administración, financiamiento y coordinación de la APS (MINSAL, 1995). Desde el punto de vista de la provisión de servicios, se ha implementado un Modelo Integral de Salud Familiar y Comunitaria, el que exhorta como pilares de su quehacer tres principios básicos: la integralidad de la atención, la continuidad del cuidado y la centralidad en las personas (Dois et al., 2016).

La Fonoaudiología por su parte, como disciplina altamente especializada (Vega et al., 2017), ha ampliado su práctica en las últimas décadas hacia el nivel primario de salud (Silva Rios, Escudero, et al., 2018). Las descripciones de la labor fonoaudiológica en salud ofrecen indicios de la incorporación profesional al primer nivel del sistema sanitario chileno (Silva Rios, Escudero, et al., 2018; Silva Rios, Rojas, et al., 2018; Tapia et al., 2016; Vega et al., 2017). Sin embargo, al observar la caracterización de los equipos profesionales que participan en
APS, es evidente la ausencia del fonoaudiólogo (Pesse-Sorensen et al., 2019).

Una reciente declaración multigremial, vinculada al reconocimiento y papel estratégico de la APS en el enfrentamiento de la pandemia, señala el papel del fonoaudiólogo respecto al seguimiento, reevaluación y rehabilitación de usuarios que presenten desórdenes de la comunicación, cognición, habla, voz y/o deglución, como consecuencias de la COVID-19 (COLMED, 2020b). Así mismo, un estudio previo destacó la importancia de acompañar a los usuarios en su proceso de salud/enfermedad, modificando la mirada clásica de la práctica fonoaudiológica y poniendo en el centro de la misma, cuestiones como el enfoque comunitario y la gestión del intersector (Tapia et al., 2016).

Lo anterior evidencia la necesidad de reflexionar respecto a los dominios de la provisión de servicios en fonoaudiología (American Speech-Language-Hearing Association [ASHA], 2016), permitiendo revisar los quehaceres genéricos y específicos del fonoaudiólogo, a la luz de los desafíos que ofrece de manera bipartita tanto la pandemia como la incorporación profesional al sector. El objetivo de esta investigación es actualizar el estado del arte respecto a los dominios de la provisión de servicios en Fonoaudiología y la pertinencia de estos en la atención primaria de la salud, a propósito de la pandemia por COVID-19.

\section{ATENCIÓN PRIMARIA DE SALUD EN LA RESPUESTA FRENTE A LA COVID-19}

La APS ha sido posicionada por la Organización Mundial de Salud (OMS) como un actor estratégico en la respuesta frente a la COVID-19, contribuyendo entre otras cosas al diagnóstico temprano, la contención psicológica y la reducción de la demanda hospitalaria (OMS, 2020). Se postula que la APS funcione como un articulador entre los diferentes niveles de atención del sistema sanitario, las organizaciones sociales e instituciones territoriales. Ello procura la continuidad de los cuidados y la mantención de programas sanitarios, así como la interrelación entre las redes que puedan sustentar respuestas ante el impacto social generado (Organización Panamericana de la Salud [OPS], 2020).

El MINSAL generó la instrucción a la APS de asumir un papel relevante en la vigilancia epidemiológica de la COVID-19, participando en el proceso de testeo, trazabilidad y aislamiento de los casos (MINSAL, 2020c). En esta línea diversas asociaciones gremiales e instituciones municipales relacionadas a la APS propusieron ejes de trabajo vinculadas al fortalecimiento del 
diagnóstico, seguimiento, trazabilidad y el robustecimiento de la red. Junto con lo anterior, se busca asegurar la continuidad de los cuidados a usuarios COVID-19 y reforzar las redes comunitarias e intersectoriales, entre otros (COLMED, 2020a).

La literatura describe el papel de la APS durante la pandemia considerando su relevancia en la contención de la propagación del virus, mediante la educación, la promoción de estrategias de cuidado a nivel individual y colectivo. Además de lo mencionado, la mantención del distanciamiento físico, la detección, el monitoreo y el aislamiento de los casos leves, contribuye a evitar la propagación del virus. También, el papel de la APS incluye la adecuación de atenciones individuales por medio de modalidad remota, la continuidad de atenciones habituales, priorizando programas de vacunación, el control de usuarios con enfermedades crónicas, el aseguramiento de atenciones a población prioritaria, entre otros. Adicionalmente, ha emergido la necesidad de articular las organizaciones, las comunidades y las personas en respuesta al impacto derivado de la pandemia por COVID-19 (Daumas et al., 2020).

Parte importante de las funciones antes descritas se desagregan en los roles y responsabilidades de los profesionales que componen los equipos, advirtiéndose un ámbito individual y uno grupal como acciones que coexisten en la APS. Es importante distinguir qué ámbitos del quehacer fonoaudiológico se vinculan con dichas acciones y cómo estos quehaceres pueden contribuir desde la disciplina a articular respuestas respecto de los procesos que se gestan en los territorios.

\section{DOMINIOS DE PROVISIÓN DE SERVICIOS DE FONOAUDIOLOGÍA}

Diversas agrupaciones gremiales de fonoaudiólogos en el mundo cuentan con descripciones detalladas del alcance de la práctica profesional, una definición oficial de la profesión y la descripción de los dominios de su quehacer (Irish Association of Speech \& Language Therapists [IASLT], 2015; New Zealand Speechlanguage Therapists' Association [NZSTA], 2012; Royal College of Speech and Language Therapists [RCSLT], 2020). En ausencia de una conceptualización definitiva del rol profesional del fonoaudiólogo en Chile, la revisión de la literatura se dirige a los dominios de provisión de servicios, ya que estos responden a aspectos que son transversales a la Fonoaudiología. Una de las principales asociaciones de patólogos del habla y del lenguaje propone 8 dominios de provisión de servicios: a) colaboración, b) asesoría, c) prevención y bienestar, d) tamizaje, e) evaluación, f) tratamiento, g) tecnología, instrumentos y modalidades y h) población y sistemas (ASHA, 2016). Por su parte, la Asociación Australiana de Patólogos del Habla, informa los siguientes servicios que provee el fonoaudiólogo: a) atención profesional independiente (desde tamizaje hasta el tratamiento), b) defensoría a usuarios, c) defensoría a través de comités o grupos de trabajo, c) asesoría, selección, prescripción, diseño y ejecución de programas de intervención, d) interpretación de instrumentos y datos para el diagnóstico, e) abordaje de conductas y generación de modificaciones ambientales que afecten la comunicación/o deglución, f) atención audiológica, g) modificación o potenciación del desempeño comunicativo y h) gestión de servicios y administración (Speech Pathology Australia [SPA], 2015). La Asociación de Habla, Lenguaje y Audiología Canadiense, por su parte, indica roles y responsabilidades profesionales como sigue: a) servicios clínicos, b) prevención, c) promoción y d) defensoría, c) educación e investigación, d) administración y e) otros (Speech-Language \& Audiology Canada [SAC], 2016).

La descripción de los puntos previos denota que las diversas asociaciones coinciden en que la práctica fonoaudiológica, si bien no está orientada a niveles específicos del sistema de salud, puede desagregarse para identificar actuaciones que aporten al sistema de salud. En esta línea, la caracterización de tópicos profesionales (ASHA, 2020b), la formulación de artículos de posicionamiento, las recomendaciones clínicas y/o declaraciones profesionales (SPA, 2020a; SAC, 2020), donde se detallan roles y responsabilidades específicas del fonoaudiólogo según el contexto de actuación profesional, han abierto la posibilidad de actualizar el papel del profesional fonoaudiólogo frente a la COVID-19. Así, se han generado documentos de posicionamiento y recomendaciones para el abordaje de los eventos relacionados con la salud secundarios a la enfermedad por coronavirus (ASHA, 2020a; SPA, 2020b). Cabe destacar que, si bien la literatura no especifica roles y responsabilidades para el fonoaudiólogo que se desempeña en APS, la exploración de los dominios de provisión de servicios permite identificar aspectos del quehacer fonoaudiológico que emergen como funciones de relevancia a desplegarse en dicho nivel del sistema sanitario, lo que implica la prevención, el bienestar, la promoción, la colaboración y la gestión. Junto con lo anterior, incluye el abordaje integral de personas afectadas por trastornos que comprometen la comunicación y/o deglución humana, entre otros. Dichos ámbitos deben analizarse con el fin de fortalecer el papel de la Fonoaudiología en Chile y ofrecer una línea de base para la formulación gremial de documentos de posición, que sean pertinentes con la discusión internacional sobre este tema. 


\section{DOMINIOS DE PROVISIÓN DE SERVICIOS DE FONOAUDIOLOGÍA DESDE EL ENFOQUE DE SALUD COMUNITARIO Y LA PROMOCIÓN DE SALUD}

El conocimiento y vínculo con las personas, familias, organizaciones y territorios que el equipo de APS sostiene (Chiara, 2016), así como la identificación de situaciones de vulnerabilidad y el trabajo intersectorial e interdisciplinario ejercido (Johansen et al., 2020), permiten al equipo articular respuestas sobre la COVID-19 considerando acciones de promoción de salud, el enfoque de salud comunitaria, las organizaciones y los líderes locales (COLMED, 2020b).

En APS las acciones correspondientes a la promoción de salud deben readecuarse y vincularse en forma consistente con la orientación del modelo integral de salud familiar y comunitaria, visibilizando los activos existentes en la comunidad para procurar la difusión de información respecto de recomendaciones frente a la COVID-19, así como fortalecer la gestión en salud junto a la comunidad. Así mismo, se destaca la necesidad de educación y apoyo a las organizaciones solidarias (COLMED, 2020b). Las acciones anteriores pueden vincularse a la provisión de servicios de Fonoaudiología, relacionada con las acciones de promoción y bienestar sociosanitario (ASHA, 2016).

En las circunstancias que rodean la crisis social y sanitaria, los profesionales de la salud insertos en APS deberán enfocarse en dar respuesta a situaciones derivadas no sólo de la patología, sino también del impacto social en las personas y comunidades (OMS, 2020). En ese sentido se destacan el aislamiento social prologando, la precarización de la vida, la violencia intrafamiliar, el consumo de drogas y el agravamiento de patologías crónicas, la necesidad de acompañamiento de las dinámicas de empoderamiento emergentes de las comunidades, así como el accionar ante los requerimientos de salud mental (Sarti et al., 2020).

Dado que la Fonoaudiología en APS mantiene acciones centradas en la rehabilitación (Silva Rios, Escudero, et al., 2018), es necesario implementar estrategias de promoción de salud individual y colectiva, con el objeto de abordar las problemáticas de la población afectada por la pandemia, posicionando la comunicación como un medio para contribuir al bienestar, el empoderamiento y el desarrollo de las personas (Mahmud et al., 2013).

Respecto a la promoción de salud, la literatura menciona la acción del fonoaudiólogo relacionada a la participación social de usuarios y usuarias con secuelas de COVID-19. Específicamente, debe contribuir a la necesidad de vinculación con diferentes nodos (personas, familia y comunidades) contemplando los intereses de la persona, posicionándose como facilitador y articulador de redes comunitarias locales. En palabras de las Sociedades Científicas y Colegios Profesionales del área de rehabilitación (2020): "insertándose en equipos inter y transdisciplinarios, considerando la complejidad social de la realidad, en especial de personas, comunidades y colectivos que presentan situación de vulneración, en los que se requiere esfuerzos mayores para el desarrollo de la acción aludida".

En el artículo de Ballesteros-Pérez \& Alfonso-Rodríguez (2019), aunque es previo a la pandemia, complementa lo anterior porque presenta una serie de definiciones respecto del dominio del fonoaudiólogo en la promoción de la salud vinculada a la población general. Se describen propuestas que aluden a la promoción de la comunicación asertiva y de la escucha activa para la autogestión comunitaria. También se exponen indicaciones para el fortalecimiento de la comunicación entendiendo que es el medio para el desarrollo de la cohesión social, inclusión, participación y empoderamiento de personas y comunidades.

Lo anterior, puede contribuir a definir el dominio relacionado con el actuar del fonoaudiólogo en la promoción de la salud comunitaria. Sin embargo, debe adecuarse al contexto contingente, entendiendo que hoy existe una fuerte amplificación de las desigualdades (Programa de las Naciones Unidas para el Desarrollo [UNDP], 2020).

En este sentido, la comunicación aparece como un factor clave (Martínez-Gómez \& Agudíez, 2012) y el fonoaudiólogo como experto en dicho ámbito (Vega et al., 2017) cumpliría un rol importante en la gestión e implementación de acciones vinculadas a la promoción de la salud, basándose en la adquisición de destrezas que impacten en un mayor control de la salud y en la efectividad comunicativa de los individuos (Ferguson \& Spence, 2012; Fernández et al., 2020).

En este punto es donde convergen las acciones profesionales del fonoaudiólogo (Hester \& Stevens-Ratchford, 2009). Ello debido que es un escenario que exige el fortalecimiento de las acciones de promoción (Paakkari \& Okan, 2020) para abordar la situación socio-sanitaria actual, desde un actuar que incluya de manera equilibrada el abordaje integral de grupos humanos, que en condiciones adversas, como es la de una pandemia, deben ser acompañados para ejercer un mayor control sobre su propia salud y alcanzar un desarrollo óptimo (Lazcano-Ponce \& AlpucheAranda, 2020). 


\section{COMENTARIOS DE LOS AUTORES}

El fonoaudiólogo que se desempeña en APS en el escenario derivado de la pandemia por COVID-19 se enfrenta a la oportunidad de ampliar el alcance de su práctica. Ello a partir del reconocimiento de la comunicación como una herramienta clave para contribuir a mejorar la salud de la población, incorporando acciones que integren la promoción de la salud a nivel individual y colectivo.

Esta revisión destaca la reflexión disciplinar y ofrece una visión integradora desde la salud pública y las declaraciones internacionales y nacionales relacionadas a los dominios de provisión de servicios de Fonoaudiología, recogiendo las recomendaciones que efectúa la OMS sobre el rol estratégico de la APS para enfrentar la crisis social y sanitaria. Asimismo, intenta dar cuenta de la pertinencia de los dominios de la Fonoaudiología en APS.

La ampliación de la provisión de servicios respecto de las prácticas clínicas habituales en contexto de pandemia, no se profundizó en esta revisión, dado que no existe una declaración sistematizada del quehacer fonoaudiológico en APS. Dicha ampliación implicará considerar las capacidades y activos en salud que presentan los ciudadanos, potenciando el bienestar general.

Entre las limitaciones de esta revisión se encuentra la ausencia de una descripción a nivel local que declare las acciones del fonoaudiólogo en APS, de acuerdo al modelo de salud integral con enfoque familiar y comunitario, así como la necesidad de contar con documentos de posición y guías que orienten a los profesionales que se desempeñan en el sector.

Como proyecciones de esta revisión emerge la necesidad de que a nivel gremial se formalice una declaración que ofrezca definiciones y lineamientos que puedan, en base a la evidencia científica disponible y a las recomendaciones de asociaciones internacionales, contribuir a delimitar el alcance de la práctica fonoaudiológica en APS. Ello contribuirá a mejorar la calidad de la provisión de servicios y orientará la formación a nivel de pre y posgrado.

Los profesionales están desafiados por tanto a identificar problemáticas y generar acciones que impliquen el acompañamiento de grupos humanos, potenciando el bienestar comunicativo, la auto-organización y la participación social, reconociendo la hibridación entre las acciones clínicas individuales y el abordaje colectivo en miras de impactar positivamente en el bienestar de la población.

\section{AGRADECIMIENTOS}

Al Fonoaudiólogo Michel Zárate Henríquez, por su contribución en el texto elaborado.

\section{REFERENCIAS}

Aguilar-Guerra, T. L., \& Reed, G. (2020). Mobilizing Primary Health Care: Cuba's Powerful Weapon against COVID-19. MEDICC Review, 22(2), 53-57.

American Speech-Language-Hearing Association [ASHA]. (2016). Scope of Practice in Speech-Language Pathology. American Speech-Language-Hearing Association; American Speech-Language-Hearing Association. https://doi.org/10.1044/policy.SP2016-00343

American Speech-Language-Hearing Association [ASHA]. (2020a). Coronavirus/COVID-19 Updates. American Speech-Language-Hearing Association; American Speech-Language-Hearing Association. https://www.asha.org/About/Coronavirus-Updates/

American Speech-Language-Hearing Association [ASHA]. (2020b). Professional Issues. American Speech-Language-Hearing Association; American SpeechLanguage-Hearing Association. https://www.asha.org/PracticePortal/Professional-Issues/

Ballesteros-Pérez, D. V., \& Alfonso-Rodríguez, L. A. (2019). El fonoaudiólogo en el ámbito de la comunidad y el bienestar social. Revista Colombiana de Rehabilitación, 18(1), 75-86. https://doi.org/10.30788/RevColReh.v18.n1.2019.327

Chiara, M. (2016). Territoriality and Health Policy: Contributions to Research and Action. Cidades. Comunidades e Territórios, 33, 1-14 https://doi.org/10.15847/citiescommunitiesterritories.dec2016.033.art01

Colegio Médico de Chile [COLMED]. (2020a). Monitorización de estrategia de Testeo-Trazabilidad-Aislamiento en la APS $\left(\mathrm{N}^{\mathrm{o}} 3\right)$. Colegio Médico de Chile. http://www.saludpublica.uchile.cl/noticias/165767/monitorizacion-de-estrategiade-testeo-trazabilidad-aislamiento-aps

Colegio Médico de Chile [COLMED]. (2020b). Reconocimiento, recursos y atribuciones para la Atención Primaria de Salud como actor estratégico en el enfrentamiento de la pandemia COVID-19 | Colegio Médico. http://www.colegiomedico.cl/reconocimiento-recursos-y-atribuciones-para-laatencion-primaria-de-salud-como-actor-estrategico-en-el-enfrentamiento-de-lapandemia-covid-19/

Cucinotta, D., \& Vanelli, M. (2020). WHO Declares COVID-19 a Pandemic. Acta Bio Medica Atenei Parmensis, 91(1), 157-160. https://doi.org/10.23750/abm.v91i1.9397

Daumas, R. P., Silva, G. A. e, Tasca, R., Leite, I. da C., Brasil, P., Greco, D. B., Grabois, V., Campos, G. W. de S., Daumas, R. P., Silva, G. A. e, Tasca, R., Leite, I. da C., Brasil, P., Greco, D. B., Grabois, V., \& Campos, G. W. de S. (2020). O papel da atenção primária na rede de atenção à saúde no Brasil: Limites e possibilidades no enfrentamento da COVID-19. Cadernos de Saúde Pública, 36(6). https://doi.org/10.1590/0102-311x00104120

Dois, A., Contreras, A., Bravo, P., Mora, I., Soto, G., \& Solís, C. (2016) Perception of users about an Integral Model of Family and Community Health care in Santiago, Chile. Revista médica de Chile, 144(5), 585-592. https://doi.org/10.4067/S0034-98872016000500005 
Europe Centre for Disease Prevention and Control [ECDC]. (2020). COVID-19 situation update worldwide, as of 16 July 2020. ECDC: European Centre for Disease Prevention and Control. https://www.ecdc.europa.eu/en/geographicaldistribution-2019-ncov-cases

Ferguson, M., \& Spence, W. (2012). Towards a definition: What does "health promotion" mean to speech and language therapists? International Journal of Language \& Communication Disorders, 47(5), 522-533. https://doi.org/10.1111/j.1460-6984.2012.00160.x

Fernández, R., Serrano, A. M., McWilliam, R. A., \& Cañadas, M. (2020). Variables predictoras del empoderamiento familiar en prácticas de atención temprana centradas en la familia. Revista de Logopedia, Foniatría y Audiología, 40(3), 128-137. https://doi.org/10.1016/j.rlfa.2020.05.003

Hester, E. J., \& Stevens-Ratchford, R. (2009). Health literacy and the role of the speech-language pathologist. American Journal of Speech-Language Pathology, 18(2), 180-191. https://doi.org/10.1044/1058-0360(2009/08-0005)

Irish Association of Speech \& Language Therapists [IASLT]. (2015). Code of Professional Conduct and Ethics. IASLT | Public Information. https://www.iaslt.ie/documents/public-information/

Johansen, A., Vracko, P., \& West, R. (2020). The evolution of community-based primary health care, Slovenia. Bulletin of the World Health Organization, 98(5), 353-359. https://doi.org/10.2471/BLT.19.239616

Lazcano-Ponce, E., \& Alpuche-Aranda, C. (2020). Alfabetización en salud pública ante la emergencia de la pandemia por Covid-19. Salud Pública de México, 62(3, may-jun), 331-340. https://doi.org/10.21149/11408

Liu, Y., Gayle, A. A., Wilder-Smith, A., \& Rocklöv, J. (2020). The reproductive number of COVID-19 is higher compared to SARS coronavirus. Journal of Travel Medicine, 27(2). https://doi.org/10.1093/jtm/taaa021

Mahmud, A. J., Olander, E., Eriksén, S., \& Haglund, B. J. (2013). Health communication in primary health care -A case study of ICT development for health promotion. BMC Medical Informatics and Decision Making, 13(1), 17. https://doi.org/10.1186/1472-6947-13-17

Martínez-Gómez, R., \& Agudíez, P. (2012). Comunicación para el Desarrollo Humano: Buscando la transformación social. CIC. Cuadernos de Información y Comunicación, 17, 79-106. https://doi.org/10.5209/rev_CIYC.2012.v17.39259

MINSAL: Establece estatuto de atención primaria de salud municipal, LEY-19378 (1995). https://www.ben.cl/leychile

Ministerio de Salud de Chile [MINSAL]. (2020a). Informe de situación COVID19. $N^{\circ}$ 16-Chile al 23 de marzo de 2020 (pp. 1-5). Departamento de Epidemiología. Ministerio de Salud de Chile. https:/www.minsal.cl/wpcontent/uploads/2020/03/Informe_10_COVID_19_Chile.pdf

Ministerio de Salud de Chile [MINSAL]. (2020b). Informe Epidemiológico $N^{\circ} 33$ Enfermedad por SARS-CoV-2 (COVID-19). Chile 13-07-2020 (pp. 1-87). Departamento de Epidemiología. Ministerio de Salud de Chile. https://www.minsal.cl/wp-content/uploads/2020/07/InformeEPI130720.pdf

Ministerio de Salud de Chile [MINSAL]. (2020c). Protocolo de coordinación para acciones de vigilancia epidemiológica durante la pandemia COVID-19 en Chile: Estrategia nacional de testeo, trazabilidad y aislamiento (pp. 1-25). Subsecretaría de Salud Pública. División de Planificación Sanitaria. Departamento de Epidemiología. https://www.minsal.cl/wp-content/uploads/2020/07/EstrategiaTesteo-Trazabilidad-y-Aislamiento.pdf
New Zealand Speech-language Therapists' Association [NZSTA]. (2012). Scope of Practice. https://speechtherapy.org.nz/wp-content/uploads/2013/09/NZSTAScope-of-Practice-2012.pdf

Organización Mundial de la Salud [OMS]. (2020). Role of primary care in the COVID-19 response (WPR/DSE/2020/004). 1-8. https://apps.who.int/iris/handle/10665/331921

Organización Panamericana de la Salud [OPS]. (2020). La atención primaria de $\begin{array}{lllll}\text { la Salud } & y & \text { la } & \text { COVID-19. }\end{array}$ https://www.campusvirtualsp.org/sites/default/files/esp_brief_report_aps_and_co vid_pdf

Paakkari, L., \& Okan, O. (2020). COVID-19: Health literacy is an underestimated problem. The Lancet Public Health, 5(5), e249-e250. https://doi.org/10.1016/S2468-2667(20)30086-4

Pesse-Sorensen, K., Fuentes-García, A., Ilabaca, J., Pesse-Sorensen, K., FuentesGarcía, A., \& Ilabaca, J. (2019). Primary Care Assessment Tool applied to primary health care workers from Conchalí, Santiago. Revista médica de Chile, 147(3), 305-313. https://doi.org/10.4067/S0034-98872019000300305

Programa de las Naciones Unidas para el Desarrollo [UNDP]. (2020). COVID-19: El desarrollo humano va camino de retroceder este año por primera vez desde 1990. UNDP. https://www.undp.org/content/undp/es/home/newscentre/news/2020/COVID19 Human_development on_course to decline for_t he_first_time_since_1990.html

Rothan, H. A., \& Byrareddy, S. N. (2020). The epidemiology and pathogenesis of coronavirus disease (COVID-19) outbreak. Journal of Autoimmunity, 109, 1-4. https://doi.org/10.1016/j.jaut.2020.102433

Royal College of Speech and Language Therapists [RCSLT]. (2020). The Royal College of Speech and Language Therapists. Speech and Language Therapy. https://www.rcslt.org/

Sarti, T. D., Lazarini, W. S., Fontenelle, L. F., Almeida, A. P. S. C., Sarti, T. D., Lazarini, W. S., Fontenelle, L. F., \& Almeida, A. P. S. C. (2020). Qual o papel da Atenção Primária à Saúde diante da pandemia provocada pela COVID-19? Epidemiologia e Serviços de Saúde, 29(2). https://doi.org/10.5123/s167949742020000200024

Sheehy, L. M. (2020). Considerations for Postacute Rehabilitation for Survivors of COVID-19. JMIR Public Health and Surveillance, 6(2), e19462. https://doi.org/10.2196/19462

Silva Rios, A., Escudero, P., Hidalgo, R., \& del Campo, M. (2018). Estudio Cualitativo de la Práctica Fonoaudiológica en el Contexto de la Atención Primaria de la Salud, en la Quinta Región de Valparaíso. Ciencia \& Trabajo, 20(62), 103106. https://doi.org/10.4067/S0718-24492018000200103

Silva Rios, A., Rojas, G., Vásquez, K., \& del Campo, M. (2018). Estudio Cualitativo de la Práctica Fonoaudiológica en el Contexto de Rehabilitación Basada en la Comunidad, en la Quinta Región de Valparaíso. Ciencia \& Trabajo, 20(63), 126-130. https://doi.org/10.4067/S0718-24492018000300126

Sociedades Científicas y Colegios Profesionales del área de rehabilitación. (2020). Consenso Interdisciplinario de Rehabilitación para Personas Adultas Post COVID-19. Sociedades Científicas y Colegios Profesionales del área de rehabilitación. https://www.socgeriatria.cl/site/?p=2668

Souza, C. D. F. de, Gois-Santos, V. T. de, Correia, D. S., Martins-Filho, P. R., Santos, V. S., Souza, C. D. F. de, Gois-Santos, V. T. de, Correia, D. S., MartinsFilho, P. R., \& Santos, V. S. (2020). The need to strengthen Primary Health Care in Brazil in the context of the COVID-19 pandemic. Brazilian Oral Research, 34. https://doi.org/10.1590/1807-3107bor-2020.vol34.0047 
Speech Pathology Australia [SPA]. (2015). Position Statements-Scope of Practice. https://www.speechpathologyaustralia.org.au/

Speech Pathology Australia [SPA]. (2020a). Clinical Guidelines. https://www.speechpathologyaustralia.org.au/SPAweb/Members/Clinical_Guidel ines/SPAweb/Members/Clinical_Guidelines/Clinical_Guidelines.aspx?hkey=0fc 81470-2d6c-4b17-90c0-ced8b0ff2a5d

Speech Pathology Australia [SPA]. (2020b). COVID-19 News and information. https://www.speechpathologyaustralia.org.au/SPAweb/About_us/SPAweb/About Us/News/COVID-19.aspx?hkey=c867dbe7-c5bc-4e16-b758-97c788ca0404

Speech-Language \& Audiology Canada [SAC]. (2016). Scope of Practice for Speech-Language Pathology. Speech-Language \& Audiology Canada. https://www.sac-oac.ca/
Speech-Language \& Audiology Canada [SAC]. (2020). Position Papers, Statements, and Guidelines. Speech-Language \& Audiology Canada. https:/www.sac-oac.ca/sac-work/position-papers-and-guidelines

Tapia, S., Espinoza, F., Herrera, P., \& Venegas, D. (2016). Caracterización de fonoaudiólogos/as insertos/as en Centros Comunitarios de Rehabilitación. Revista Chilena de Fonoaudiología, 15, 1-13. https://doi.org/10.5354/rcdf.v15i0.44186

Vega, Y. E., Torres, A. M., \& del Campo, M. N. (2017). Análisis del Rol del Fonoaudiólogo(a) en el Sector Salud en Chile. Ciencia \& trabajo, 19(59), 76-80. https://doi.org/10.4067/S0718-24492017000200076 